\title{
Plumbagin attenuates high glucose-induced trophoblast cell apoptosis and insulin resistance via activating AKT/mTOR pathway
}

\author{
Yilin Zhang ${ }^{1}$, Guantai $\mathrm{Ni}^{1}$, Hongying Yang ${ }^{2 *}$ \\ ${ }^{1}$ Department of Obstetrics and Gynecology, The First Affiliated Hospital of Wannan Medical College, Wuhu, Anhui, \\ 241001, China; ${ }^{2}$ Department of Obstetrics, The Ninth People's hospital of Chongqing, Chongqing, 400700, China
}

"Corresponding author: Hongying Yang, Department of Obstetrics,The Ninth People's hospital of Chongqing, No. 69, Jialing Village, Beibei District, Chongqing City, China. Email: yanghongying_666@163.com

Received: 7 Sepember 2021; Accepted: 10 October 2021; Published: 6 November 2021

(c) 2021 Codon Publications

OPEN ACCESS (c) (1) (2) (2)

ORIGINAL ARTICLE

\begin{abstract}
Plumbagin, a bioactive phytoconstituent, is isolated from the root of Plumbago zeylanica L. Plumbagin possesses antidiabetic effect to mediate glucose homeostasis, wound healing and diabetic nephropathy. However, the involvement of plumbagin in gestational diabetes mellitus (GDM) has not been reported yet. Trophoblast cell line (HTR8/SVneo) was incubated with high glucose to establish cell model of GDM. Cell viability and proliferation were detected by MTT and EdU staining. Flow cytometry was used to investigate cell apoptosis. Cell viability of HTR8/SVneo was reduced by high glucose or incubation of plumbagin. Plumbagin restored reduced cell viability and proliferation of HTR8/SVneo induced by high glucose. Plumbagin attenuated high glucose-induced cell apoptosis in HTR8/SVneo cells through upregulation of Bcl-2 and down-regulation of Bax, cleaved caspase-3 and cleaved caspase-9. Protein expression of glucose transporter type 4 (GLUT-4), insulin receptor (INSR)-B and INSR substrate (IRS1) was decreased in high glucose-induced HTR8/SVneo but increased by plumbagin. The suppressive effects of high glucose on phosphorylation of AKT and mTOR in HTR8/SVneo were reversed by plumbagin. Plumbagin improved high glucose-induced cell apoptosis and insulin resistance of HTR8/SVneo through activation of AKT/mTOR pathway, suggesting that plumbagin might be used as a potential strategy for the prevention of GDM.
\end{abstract}

Keywords: plumbagin; high glucose; trophoblast cell; insulin resistance; AKT/mTOR

\section{Introduction}

Gestational diabetes mellitus (GDM) is a disease that occurs during pregnancy, usually accompanied by hyperglycemia and insulin resistance, which seriously affects the health of mother and baby (Agarwal, 2016). The incidence of GDM in China increased rapidly and reached about 15\% (Yan et al., 2019). GDM usually causes a series of serious metabolic diseases, such as type 2 diabetes mellitus, abnormal fetal development, abortion and fetal asphyxia (Malaeb et al., 2020; Vince et al., 2020). Dietary or pharmacological interventions have been used for the prevention of GDM (Tanase-Nakao et al., 2017). Early diagnosis and effective prevention are still urgent for patients with GDM.

The placenta functions as an organ for blood circulation, nutrition and exchange of gases between mother and fetus during pregnancy, and ensures a healthy pregnancy and embryonic development (Kaiser, 2014). Abnormal functioning of the placenta is associated with adverse pregnancy outcomes caused by GDM (Ilekis et al., 2016). During development of GDM, the placenta secrets hormones to enhance insulin resistance and antagonize insulin, thus promoting GDM (Tang et al., 2020). In addition, GDM also leads to placenta dysplasia 
and vascular abnormalities to aggravate progression of GDM (Madazlı et al., 2008). Therefore, regulation of placental function is important for amelioration of GDM. Trophoblastic cells are vital for the development of the placenta through differentiation, invasion and migration to vascular tissue (Chang and Vivian Yang, 2013). A study has demonstrated that high glucose induces impaired the proliferation, invasion and migration activities of trophoblast cells, leading to dysplasia of the placental tissue, and resulting in abortion, spontaneous abortion and premature birth (Chang and Vivian Yang, 2013). Strategy to mediate high glucose-induced trophoblast cell dysfunction was established as an effective treatment for GDM.

Plumbagin, isolated from the root of Plumbago zeylanica L., is a naphthoquinone derivative, and exerts antioxidant, anti-inflammatory, antiviral, anti-tumor and neuroprotective effects. For example, plumbagin promotes reactive oxygen species, apoptosis and autophagy of cancer cells while suppressing cell proliferation and survival (Yin et al., 2020). Plumbagin modulates neuro-inflammatory and apoptotic pathways to attenuate cerebral ischemia (Chen et al., 2018). Plumbagin also regulates oxidative stress, lipid inflammation and metabolism to reduce nonalcoholic fatty liver disease and obesity (Pai et al., 2019). In addition, plumbagin promoted expression of glucose transporter-4 to contribute to glucose homeostasis of streptozotocin-induced diabetic rats (Sunil et al., 2012), and ameliorated diabetic nephropathy (Yong et al., 2013) and wound healing (Shao et al., 2019) in diabetic rats. However, the role and mechanism of plumbagin in GDM are rarely reported.

In this study, high glucose-induced trophoblast cell line (HTR8/SVneo) was established, and the effects of plumbagin on cell proliferation, apoptosis and insulin resistance of HTR8/SVneo were investigated. Therefore, meaningful results could provide potential strategy for the prevention of GDM.

\section{Materials and methods}

\section{Cell culture and treatment}

HTR8/SVneo cells were purchased from Jining Cell Culture Center (Shanghai, China) and cultured in RPMI 1640 medium containing 1\% streptomycin-penicillin and $10 \%$ fetal bovine serum (Thermo Fisher Scientific, Waltham, CA, USA) in a humidified incubator at $37^{\circ} \mathrm{C}$. Cells were treated with 5-, 10-, 20-, 40- or 80- $\mu \mathrm{M}$ plumbagin (Sigma-Aldrich, St Louis, LA, USA) for $24 \mathrm{~h}$ before MTT colorimetric assay. Cells were also treated with 5.5-, 12.5-, 25- or 50-mM glucose for $24 \mathrm{~h}$ before MTT assay. In line with previous study that in vitro cell model of GDM was established through incubation with $25-\mathrm{mM}$ glucose (Zhou, Xiang, \& Zheng, 2019), HTR8/SVneo cells were treated with $25-\mathrm{mM}$ glucose in the presence of $5-, 10-$ and $20-\mu \mathrm{M}$ plumbagin for $24 \mathrm{~h}$.

\section{Cell viability and proliferation assays}

HTR8/SVneo cells with indicated treatment were plated in a 96-well plate for $48 \mathrm{~h}$. The MTT solution (SigmaAldrich) was added to the well. Cells were cultured for $4 \mathrm{~h}$, and incubated with $100-\mu \mathrm{L}$ dimethyl sulfoxide (DMSO) for $10 \mathrm{~min}$. Optical density at $490 \mathrm{~nm}$ was measured on microplate reader (Bioteck, Winooski, VT, USA). For incorporation of 5-ethynyl-2'-deoxyuridine (EdU), HTR8/SVneo cells with indicated treatment were plated in a 96-well plate and incubated with $50-\mu \mathrm{M}$ EdU (RiboBio, Guangzhou, China) for $12 \mathrm{~h}$. Cells were fixed with $4 \%$ paraformaldehyde, incubated with $2-\mathrm{mg} / \mathrm{mL}$ glycine followed by $0.5 \%$ Triton X-100. EdU antibody from EdU Cell Proliferation Detection (RiboBio) was added and immunostaining was performed with Apollo staining reaction buffer. Cells were measured under fluorescence microscopy (Nikon, Tokyo, Japan) followed by staining with DAPI (4',6-diamidino-2-phenylindole fluorescent stain).

\section{Cell apoptosis assay}

HTR8/SVneo cells with indicated treatment were harvested and resuspended with $1 \times$ buffer from FITC Annexin V Apoptosis Detection Kit (BD Biosciences, Franklin Lakes, NJ, USA). The cell suspension was then incubated with Annexin V-fluorescein isothiocyanate and propidium iodide before analyzed by FACSCanto $\mathrm{II}^{\mathrm{TM}}$ flow cytometry system (BD Biosciences, Franklin Lakes, NJ, USA).

\section{Western blot}

Proteins were isolated from cultured HTR8/SVneo cells through RIPA lysis buffer (Beyotime, Beijing, China), and electrophoresed on sodium dodecyl sulfate-polyacrylamide gel electrophoresis. The membranes were blocked with 5\% skim milk after transferred to polyvinylidene fluoride membranes (Millipore, Billerica, MA, USA). The membranes were incubated with the following specific primary antibodies: anti-cleaved caspase- 3 and anticleaved caspase-9 (1:2,000; Cell Signaling, Boston, MA, USA), anti-Bax and anti-Bcl2 (1:2,500; Abcam, Shanghai, China), anti-GLUT4 and anti-INSR-B (1:3,000; Cell Signaling), anti-IRS1 and anti- $\beta$-actin (1:3,500; Abcam), anti-AKT and anti-p-AKT (1:4,000; Cell Signaling), anti-mTOR and anti-p-mTOR (1:4,500; Abcam). The immunoreactive signals were measured by enhanced 
chemiluminescence reagent kit (Millipore) followed by incubation with the corresponding secondary antibodies.

\section{Statistical analysis}

All experimental data at least in triplicate were manifested as mean \pm standard deviation. Differences between groups were analyzed by Student's $t$-test or one-way analysis of variance under the Graphpad Prism 7 software (GraphPad Inc., La Jolla, CA, USA). $P<0.05$ was established as statistically significant.

\section{Results}

\section{Plumbagin promoted cell proliferation of high glucose induced HTR8/SVneo}

In vitro cell model of GDM was established through incubation of HTR8/SVneo cells with high glucose. High glucose reduced cell viability of HTR8/SVneo in a dose-dependent manner (Figure 1A). Plumbagin also reduced the cell viability of HTR8/SVneo in a dosagedependent manner (Figure 1B). Less than 20- $\mu \mathrm{M}$ plumbagin had no significant effect on the cell viability of HTR8/SVneo (Figure 1B). Therefore, high glucoseinduced HTR8/SVneo cells were incubated with 5-, 10- or $20-\mu \mathrm{M}$ plumbagin to investigate the effects of plumbagin on GDM. Plumbagin enhanced the cell viability of high glucose-induced HTR8/SVneo (Figure 1C), and promoted cell proliferation (Figure 1D) with increased EdUpositive cells (Figure 1E). These results demonstrated the proliferative effect of plumbagin on GDM.

\section{Plumbagin repressed cell apoptosis of high glucose-induced HTR8/SVneo}

High glucose-induced cell apoptosis of HTR8/SVneo cells (Figure 2A), and plumbagin incubation repressed the apoptosis of high glucose-induced HTR8/SVneo (Figure 2A). Moreover, reduced $\mathrm{Bcl}-2$ and enhanced Bax, and cleaved caspase- 3 and cleaved caspase-9, in high glucose-induced HTR8/SVneo cells were restored by plumbagin incubation (Figure 2B), suggesting the anti-apoptotic role of plumbagin in GDM.

\section{Plumbagin improved insulin resistance of high glucose-induced HTR8/SVneo}

High glucose decreased protein expression of GLUT4, INSR-B and IRS1 in HTR8/SVneo cells (Figure 3). However, the levels of insulin-signaling and glucose utilization-associated factors, GLUT-4, INSR-B and IRS1, in high glucose-induced HTR8/SVneo were restored by plumbagin (Figure 3), indicating that plumbagin improved the insulin response of trophoblast cells during the development of GDM.

\section{Plumbagin promoted activation of AKT/mTOR in high glucose-induced HTR8/SVneo}

High glucose or plumbagin incubation had no significant effects on the protein expression of AKT and mTOR in HTR8/SVneo (Figure 4). High glucose reduced protein expression of AKT and mTOR phosphorylation in HTR8/SVneo (Figure 4). Plumbagin attenuated high glucose-induced decrease of AKT and mTOR phosphorylation in HTR8/SVneo (Figure 4), revealing that plumbagin promoted activation of AKT/mTOR to ameliorate GDM.

\section{Discussion}

Naphthoquinones are phytochemicals isolated from medicinal plants that possess pharmacological potential of antidiabetic activity through increasing glucose uptake and inhibition of protein tyrosine phosphatase 1B and $\alpha$-glucosidase (Shah et al., 2018). Naphthoquinone-based hybrids exert antagonistic activity against type-2 diabetes (Paul et al., 2019). Plumbagin has been depicted as a naphthoquinone derivative that can reduce blood glucose to attenuate streptozotocin-induced diabetes (Sunil et al., 2012). The antidiabetic effect of plumbagin on GDM has been investigated in this study.

The placenta is considered a target organ of GDM with ability to maintain immunologic, endocrine and nutritional functions between mother and fetus (CarrascoWong et al., 2020). Hyperglycemia in mother alters structure of the placenta and facilitates fetus malformation and abortion during the development of GDM (Carrasco-Wong et al., 2020). Functions of trophoblast cells are key regulator of placenta development, and the abnormal growth, migration and invasion are implicated in placenta maldevelopment (Zhang et al., 2021). High glucose, with ability to induce impairment in the proliferation, invasion and migration activities of trophoblast cells, has been widely used for the establishment of in vitro cell model of GDM (Zhang et al., 2021). Results in this study demonstrated that high glucose reduced cell viability of HTR8/SVneo, suppressed cell proliferation and promoted cell apoptosis. Moreover, suppression of high glucose-induced biological dysfunction of trophoblast cells facilitated for the amelioration of GDM (Zhang et al., 2021). Plumbagin incubation restored the cell viability of high glucose-induced HTR8/SVneo, promoted cell proliferation and suppressed cell apoptosis, suggesting anti-apoptotic and proliferative effects against GDM. 


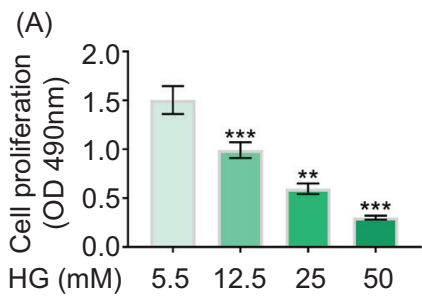

(D)
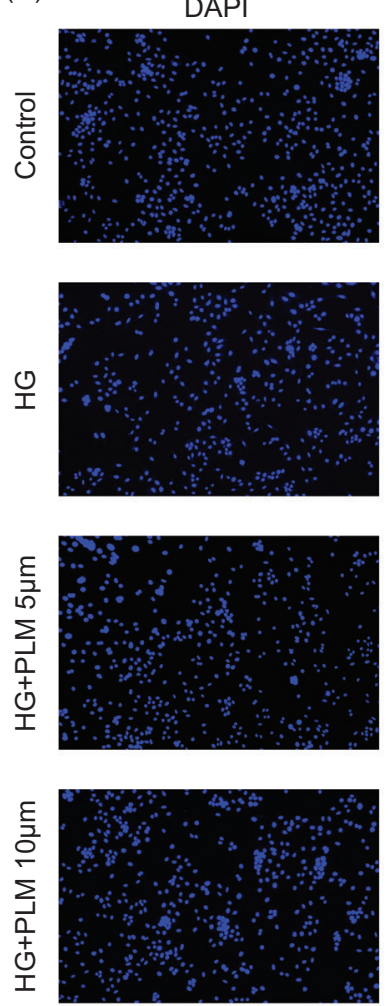

(E)

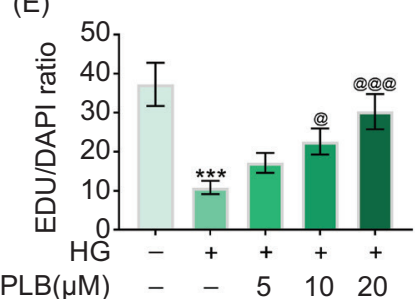

(B)

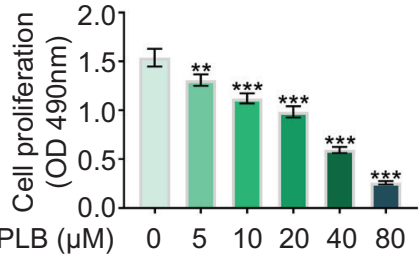

(C)

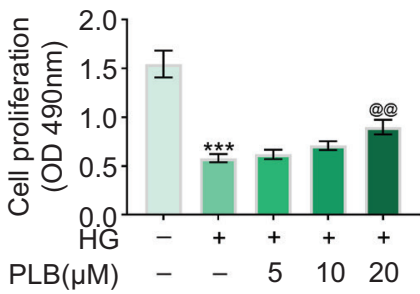

$\begin{array}{lll}5 & 10 & 20\end{array}$

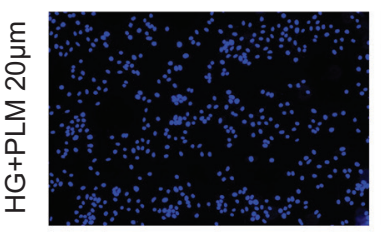

MERGE
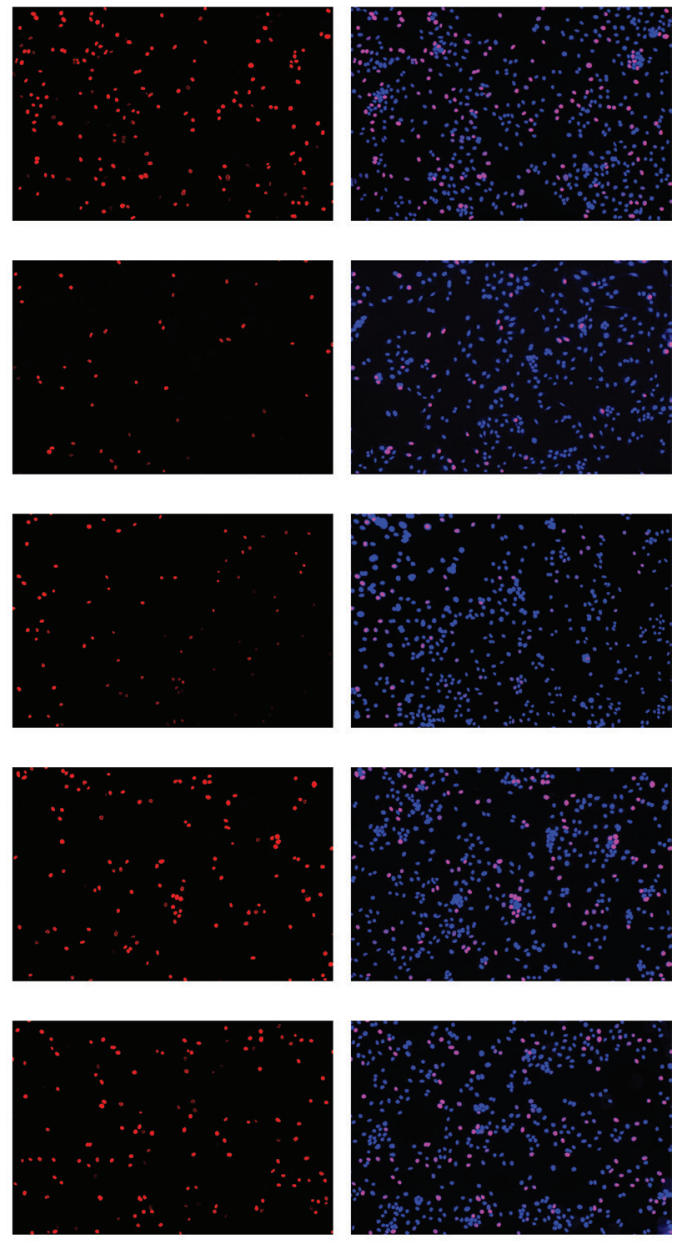

Figure 1. Plumbagin promoted cell proliferation of high glucose-induced HTR8/SVneo. (A) High glucose reduced cell viability of HTR8/SVneo in a dose-dependent manner. (B) Plumbagin reduced HTR8/SVneo cell viability in a dose-dependent manner. (C) Plumbagin enhanced cell viability of high glucose-induced HTR8/SVneo. (D) Plumbagin promoted the cell proliferation of high glucose-induced HTR8/SVneo. (E) Plumbagin promoted EdU-positive cells of high glucose-induced HTR8/SVneo. @ $P<0.05$; ${ }^{* *}$, @@P $<0.01 ;{ }^{* * *}$, @@@ $P<0.001$.

In addition, migration and invasion of trophoblasts were also implicated in the pathogenesis of GDM (Sun et al., 2020), and plumbagin exerted anti-invasive effect against breast cancer cells (Lee et al., 2012). The effects of plumbagin on cell migration and invasion of high glucose-induced HTR8/SVneo cells must be investigated in the future research.

During the development of GDM, insulin signal transduction and glucose metabolism in the placenta were disturbed, leading to pathological changes in the placenta tissue (Peng et al., 2020). Increase in insulin sensitivity is established as strategy to improve GDM (Peng et al., 2020). Plumbagin has been reported to regulate GLUT4 translocation to reduce blood glucose, and attenuate streptozotocin-induced diabetes (Sunil et al., 2012). Results of this study demonstrated that plumbagin increased protein expression of GLUT4 and insulin signaling molecules, including IRS1 and INSR-B, in high glucose-induced HTR8/SVneo, thus promoting insulin sensitivity and suppressing insulin resistance to protect against GDM. Imbalance of inflammation in the placenta with increase in pro-inflammatory cytokines and decrease in anti-inflammatory cytokines mediated proliferation and invasion of trophoblasts and activities of insulin and glucose receptors, thus contributing to the pathogenesis of GDM (Li et al., 2021). Therefore, the anti-inflammatory effect of plumbagin on high glucose-induced HTR8/SVneo must also be investigated in the future studies.

A previous study has demonstrated that AKT/mTOR pathway was involved in the development of GDM through regulation of insulin resistance and trophoblastic 

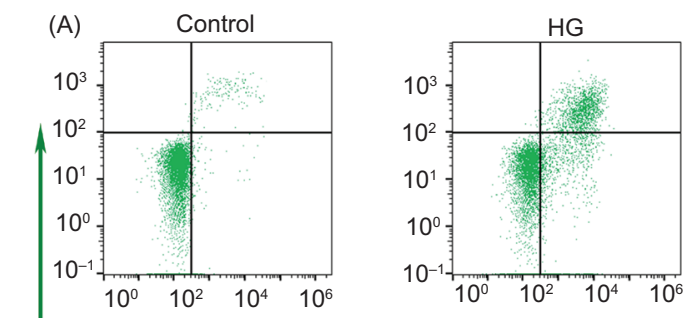

(B)
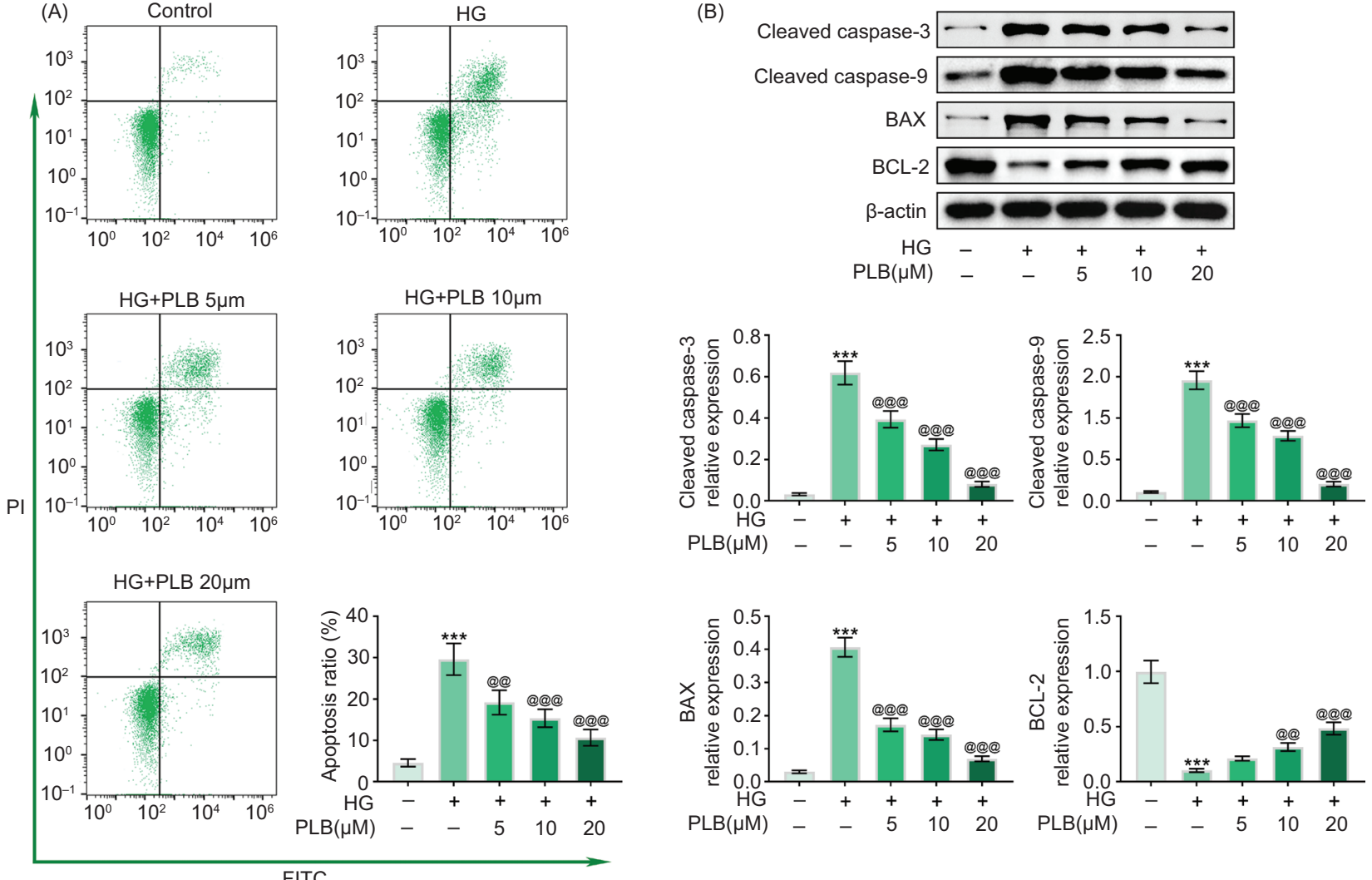

Figure 2. Plumbagin repressed cell apoptosis of high glucose-induced HTR8/SVneo. (A) Plumbagin suppressed the cell apoptosis of high glucose-induced HTR8/SVneo. (B) Plumbagin attenuated high glucose-induced decrease of Bcl-2, increase of Bax, cleaved caspase-3, and cleaved caspase-9 in HTR8/SVneo. @@P $<0.01$; ${ }^{*+*}$,@@ $P<0.001$.
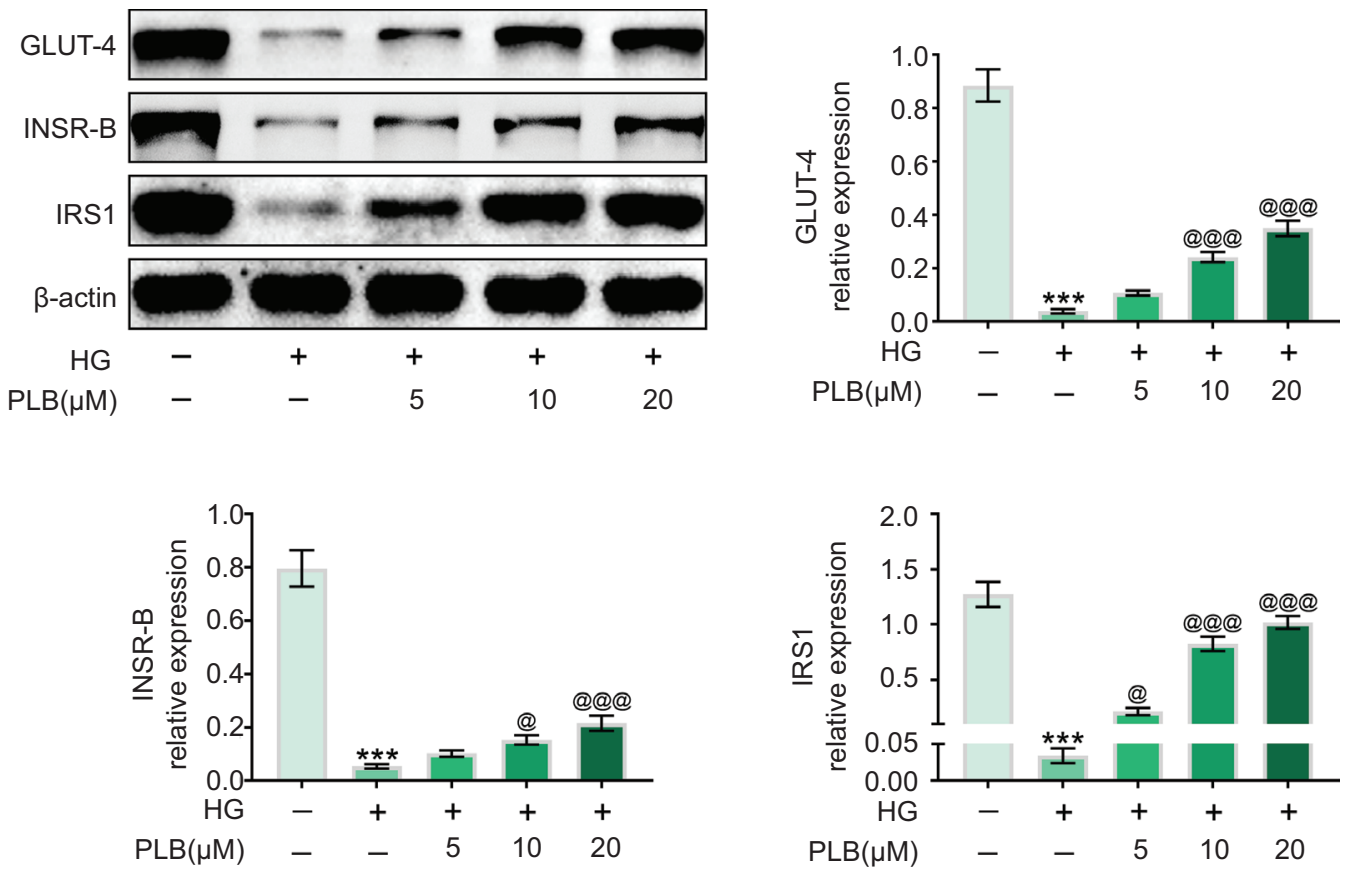

Figure 3. Plumbagin improved insulin resistance of high glucose-induced HTR8/SVneo Plumbagin attenuated high glucose-induced decrease of GLUT-4, INSR-B and IRS1 in HTR8/SVneo cells. @P $<0.05 ; "$, @@@P $<0.001$. 

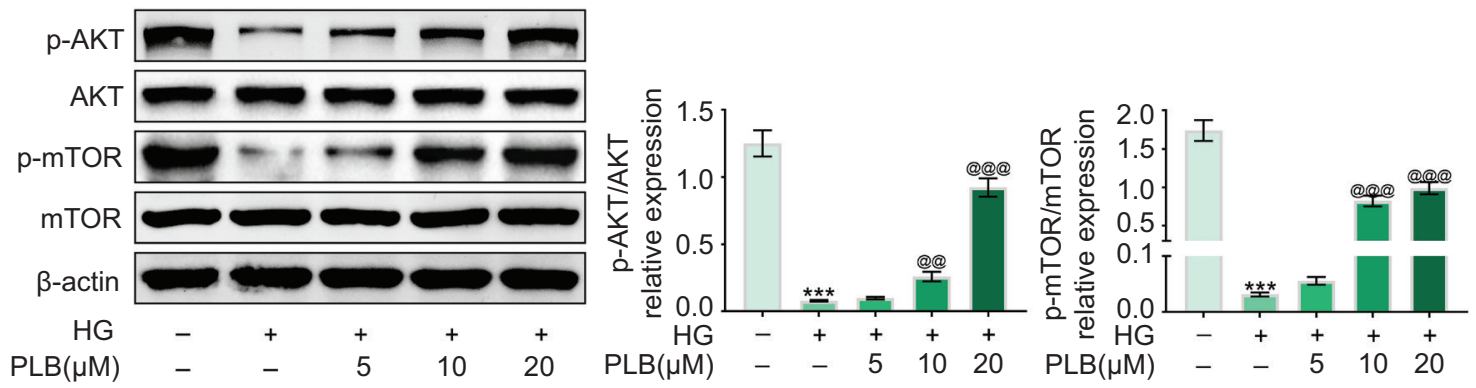

Figure 4. Plumbagin promoted activation of AKT/mTOR in high glucose-induced HTR8/SVneo. Plumbagin attenuated high glucose-induced decrease of AKT and mTOR phosphorylation in HTR8/SVneo. @@ $P<0.01$; "*t, @@@P $<0.001$.

function (Tsai et al., 2021). High glucose induced down-regulation of mTOR phosphorylation in trophoblasts (Tsai et al., 2021), and activation of AKT/mTOR impeded the progression of GDM (Han et al., 2020). Therefore, regulation of AKT/mTOR pathway provided avenues to alleviate GDM. PI3K/Akt/mTOR was implicated in the plumbagin-mediated cancer cell proliferation and survival (Cai et al., 2020) and neuroprotection (Yuan et al., 2017). Here, the protein expression of AKT and mTOR phosphorylation was reduced in high glucose-induced HTR8/SVneo but enhanced by plumbagin incubation, indicating that plumbagin promoted activation of AKT/mTOR pathway to attenuate GDM.

\section{Conclusion}

Plumbagin promoted cell proliferation of high glucose-induced HTR8/SVneo cells, suppressed cell apoptosis and promoted insulin resistance through activation of AKT/mTOR pathway. These results could provide a novel therapeutic strategy for treating GDM.

\section{References}

Agarwal, M.M., 2016. Gestational diabetes mellitus: screening with fasting plasma glucose. World Journal of Diabetes 7: 279-289. https://doi.org/10.4239/wjd.v7.i14.279

Cai, Z., He, S., Li, T., Zhao, L. and Zhang, K., 2020. Plumbagin inhibits proliferation and promotes apoptosis of ovarian granulosa cells in polycystic ovary syndrome by inactivating PI3K/ Akt/mTOR pathway. Animal Cells and Systems 24: 197-204. https://doi.org/10.1080/19768354.2020.1790416

Carrasco-Wong, I., Moller, A., Giachini, F.R., Lima, V.V., Toledo, F., Stojanova, J., Sobrevia, L. and San Martín, S., 2020. Placental structure in gestational diabetes mellitus. Biochimica et Biophysica Acta (BBA) - Molecular Basis of Disease 1866: 165535. https://doi.org/10.1016/j.bbadis.2019.165535

Chang, S.-C. and Vivian Yang, W.-C., 2013. Hyperglycemia induces altered expressions of angiogenesis associated molecules in the trophoblast. Evidence-Based Complementary and
Alternative Medicine: eCAM 2013: 457971-457971. https://doi. org/10.1155/2013/457971

Chen, X.-J., Zhang, J.-G. and Wu, L., 2018. Plumbagin inhibits neuronal apoptosis, intimal hyperplasia and also suppresses TNF- $\alpha /$ NF- $\mathrm{kB}$ pathway-induced inflammation and matrix metalloproteinase-2/9 expression in rat cerebral ischemia. Saudi Journal of Biological Sciences 25: 1033-1039. https://doi.org/10.1016/j. sjbs.2017.03.006

Han, N., Fang, H.-Y., Jiang, J.-X. and Xu, Q., 2020. Downregulation of microRNA-873 attenuates insulin resistance and myocardial injury in rats with gestational diabetes mellitus by upregulating IGFBP2. American Journal of Physiology-Endocrinology and Metabolism 318: E723-E735. https://doi.org/10.1152/ ajpendo.00555.2018

Ilekis, J.V., Tsilou, E., Fisher, S., Abrahams, V.M., Soares, M.J., Cross, J.C., Zamudio, S., Illsley, N.P., Myatt, L., Colvis, C., Costantine, M.M., Haas, D.M., Sadovsky, Y., Weiner, C., Rytting, E. and Bidwell, G., 2016. Placental origins of adverse pregnancy outcomes: potential molecular targets: an Executive Workshop Summary of the Eunice Kennedy Shriver National Institute of Child Health and Human Development. American Journal of Obstetrics and Gynecology 215: S1-S46. https://doi. org/10.1016/j.ajog.2016.03.001

Kaiser, J., 2014. Gearing up for a closer look at the human placenta. Science 344: 1073. https://doi.org/10.1126/ science.344.6188.1073

Lee, J.-H., Yeon, J.-H., Kim, H., Roh, W., Chae, J., Park, H.-O. and Kim, D.-M., 2012. The natural anticancer agent plumbagin induces potent cytotoxicity in MCF-7 human breast cancer cells by inhibiting a PI-5 kinase for ROS generation. PloS One 7: e45023. https://doi.org/10.1371/journal.pone.0045023

Li, J., Li, Y., Zhou, X., Wei, L., Zhang, J., Zhu, S., Zhang, H., Gao, X., Sharifu, L.M., Wang, S., Xi, L. and Feng, L., 2021. Upregulation of IL-15 in the placenta alters trophoblasts behavior contributing to gestational diabetes mellitus. Cell \& Bioscience 11: 33. https://doi.org/10.1186/s13578-021-00533-4

Madazlı, R., Tuten, A., Calay, Z., Uzun, H., Uludag, S. and Ocak, V., 2008. The incidence of placental abnormalities, maternal and cord plasma malondialdehyde and vascular endothelial growth factor levels in women with gestational diabetes mellitus and nondiabetic controls. Gynecologic and Obstetric Investigation 65: 227-232. https://doi.org/10.1159/000113045 
Malaeb, D., Hallit, S., Sacre, H., Hallit, R. and Salameh, P., 2020. Factors associated with wheezing among Lebanese children: results of a cross-sectional study. Allergologia et Immunopathologia 48: 523-529. https://doi.org/10.1016/j. aller.2020.02.003

Pai, S.A., Munshi, R.P., Panchal, F.H., Gaur, I.-S., Mestry, S.N., Gursahani, M.S. and Juvekar, A.R., 2019. Plumbagin reduces obesity and nonalcoholic fatty liver disease induced by fructose in rats through regulation of lipid metabolism, inflammation and oxidative stress. Biomedicine \& Pharmacotherapy 111: 686694. https://doi.org/10.1016/j.biopha.2018.12.139

Paul, A., Viswanathan, G.K., Mahapatra, S., Balboni, G., Pacifico, S., Gazit, E. and Segal, D., 2019. Antagonistic activity of naphthoquinone-based hybrids toward amyloids associated with Alzheimer's disease and type- 2 diabetes. ACS Chemical Neuroscience 10: 3510-3520. https://doi.org/10.1021/acschemneuro. 9b00123

Peng, H.-Y., Li, H.-P. and Li, M.-Q., 2020. Downregulated ABHD5 aggravates insulin resistance of trophoblast cells during gestational diabetes mellitus. Reproductive Sciences 27: 233-245. https://doi.org/10.1007/s43032-019-00010-x

Shah, M.A., Keach, J.E. and Panichayupakaranant, P., 2018. Antidiabetic naphthoquinones and their plant resources in Thailand. Chemical and Pharmaceutical Bulletin 66: 483-492. https://doi.org/10.1248/cpb.c17-00529

Shao, Y., Dang, M., Lin, Y. and Xue, F., 2019. Evaluation of wound healing activity of plumbagin in diabetic rats. Life Sciences 231: 116422. https://doi.org/10.1016/j.lfs.2019.04.048

Sun, D.-G., Tian, S., Zhang, L., Hu, Y., Guan, C.-Y., Ma, X. and Xia, H.-F., 2020. The miRNA-29b Is downregulated in placenta during gestational diabetes mellitus and may alter placenta development by regulating trophoblast migration and invasion through a HIF3A-dependent mechanism. Frontiers in Endocrinology 11: 169. https://doi.org/10.3389/fendo.2020.00169

Sunil, C., Duraipandiyan, V., Agastian, P. and Ignacimuthu, S., 2012. Antidiabetic effect of plumbagin isolated from Plumbago zeylanica L. root and its effect on GLUT4 translocation in streptozotocin-induced diabetic rats. Food and Chemical Toxicology 50: 4356-4363. https://doi.org/10.1016/j.fct.2012.08.046

Tanase-Nakao, K., Arata, N., Kawasaki, M., Yasuhi, I., Sone, H., Mori, R. and Ota, E., 2017. Potential protective effect of lactation against incidence of type 2 diabetes mellitus in women with previous gestational diabetes mellitus: a systematic review and meta-analysis. Diabetes/Metabolism Research and Reviews 33: e2875. https://doi.org/10.1002/dmrr.2875

Tang, L., Li, P. and Li, L., 2020. Whole transcriptome expression profiles in placenta samples from women with gestational diabetes mellitus. Journal of Diabetes Investigation 11: 1307-1317. https://doi.org/10.1111/jdi.13250

Tsai, K., Tullis, B., Jensen, T., Graff, T., Reynolds, P. and Arroyo, J., 2021. Differential expression of mTOR related molecules in the placenta from gestational diabetes mellitus (GDM), intrauterine growth restriction (IUGR) and preeclampsia patients. Reproductive Biology 21: 100503. https://doi.org/10.1016/j. repbio.2021.100503

Vince, K., Perković, P. and Matijević, R., 2020. What is known and what remains unresolved regarding gestational diabetes mellitus (GDM). Journal of Perinatal Medicine 48: 757-763. https://doi. org/10.1515/jpm-2020-0254

Yan, B., Yu, Y., Lin, M., Li, Z., Wang, L., Huang, P., Song, H., Shi, X., Yang, S., Li, X. and Li, X., 2019. High, but stable, trend in the prevalence of gestational diabetes mellitus: a population-based study in Xiamen, China. Journal of Diabetes Investigation 10: 1358-1364. https://doi.org/10.1111/jdi.13039

Yin, Z., Zhang, J., Chen, L., Guo, Q., Yang, B., Zhang, W. and Kang, W., 2020. Anticancer Effects and Mechanisms of Action of Plumbagin: review of research advances. BioMed Research International 2020: 6940953-6940953. https://doi.org/10.1155/2020/6940953

Yong, R., Chen, X.-M., Shen, S., Vijayaraj, S., Ma, Q., Pollock, C.A. and Saad, S., 2013. Plumbagin ameliorates diabetic nephropathy via interruption of pathways that include NOX4 signalling. PloS one 8: e73428. https://doi.org/10.1371/journal.pone.0073428

Yuan, J.-H., Pan, F., Chen, J., Chen, C.-E., Xie, D.-P., Jiang, X.-Z., Guo, S.-J. and Zhou, J., 2017. Neuroprotection by plumbagin involves BDNF-TrkB-PI3K/Akt and ERK1/2/JNK pathways in isoflurane-induced neonatal rats. Journal of Pharmacy and Pharmacology 69: 896-906. https://doi.org/10.1111/jphp.12681

Zhang, L., Zeng, M., Tang, F., Chen, J., Cao, D. and Tang, Z.-N., 2021. Circ-PNPT1 contributes to gestational diabetes mellitus (GDM) by regulating the function of trophoblast cells through miR-889-3p/PAK1 axis. Diabetology \& Metabolic Syndrome 13: 58-58. https://doi.org/10.1186/s13098-021-00678-9

Zhou, X., Xiang, C. and Zheng, X., 2019. miR-132 serves as a diagnostic biomarker in gestational diabetes mellitus and its regulatory effect on trophoblast cell viability. Diagnostic pathology 14: $1-7$. 